\title{
On Efficient Meta-Data Collection for Crowdsensing
}

\author{
Luke Dickens \\ Department of Computing \\ Imperial College London \\ Email: luke.dickens@imperial.ac.uk
}

\author{
Emil Lupu \\ Department of Computing \\ Imperial College London \\ Email: e.c.lupu@imperial.ac.uk
}

\begin{abstract}
Participatory sensing applications have an on-going requirement to turn raw data into useful knowledge, and to achieve this, many rely on prompt human generated meta-data to support and/or validate the primary data payload. These human contributions are inherently error prone and subject to bias and inaccuracies, so multiple overlapping labels are needed to cross-validate one another. While probabilistic inference can be used to reduce the required label overlap, there is still a need to minimise the overhead and improve the accuracy of timely label collection. We present three general algorithms for efficient human meta-data collection, which support different constraints on how the central authority collects contributions, and three methods to intelligently pair annotators with tasks based on formal information theoretic principles. We test our methods' performance on challenging synthetic data-sets, based on real data, and show that our algorithms can significantly lower the cost and improve the accuracy of human meta-data labelling, with little or no impact on time.
\end{abstract}

\section{INTRODUCTION}

Many participatory sensing (PS), or crowdsensing, systems require regular human input, to translate sensing data into meaningful information. However, human input is fundamentally unreliable (semi-trusted). This paper proposes novel architectures and algorithms, that utilise recent trust based inference techniques [1], [2], to flexibly maximise throughput of valuable human input within crowdsensing systems.

PS applications are becoming increasingly popular and complex, and can be used as direct data sources or for processing pipelines [3], [4]. PS has been used to sense, measure and map a variety of phenomena, including: individuals' health, mobility \& social status, e.g. [5]-[7]; fuel \& grocery prices, e.g. [8], [9]; air quality \& pollution levels [3], [10], [11]; biodiversity [12]; transport infrastructure, e.g. [13]-[15]; and route-planning for drivers [16] \& cyclists [17].

There are various conditions under which meta-data based on human acquired labels are used in PS applications. For instance, when data is shared directly with participants, such as the Biketastic scheme from [17], where users share cycle routes and associated information. Here, route information is a combination of data from sensors (accelerometers, GPS, microphones,...), with human generated content such as location tags, photos of interesting features, and the implicit information that this a good route. For optimal user experience, only the best routes should be shared, with poor routes or incorrect information weeded out. Other direct sharing applications with similar requirements include environmental sensing in [12] and social sensing in [5]. Some applications instead collect and improve labelled data-sets for use with automated systems, such as users tagging map locations in [5], [8] to be used on mapping services, e.g. Wikimapia. Here application and service have a mutually beneficial relationship, and both rely on the quality of the human tags.

In other cases, human intervention is required to validate sensed data, e.g. manual calibration of phone sensors is required for noise measurements in [11], and air-quality data in [3]. Validation can also be required for meta-data, either when the meta-data has stand-alone utility, like the map locations in [5], or when it is used as training data for machine learning inference, e.g. the pot-hole detector from [14] and the activity recognition from [7]. Finally, these applications provide data that could be commercially valuable [4], e.g. health data to pharmaceutical companies, social data to advertisers and so on, which can put formal Quality of Information (QoI) demands on the data and meta-data.

These human contributions can be error prone, inaccurate and/or biased. Therefore, this paper exploits probabilistic models for inference with semi-trusted labels, e.g. [1], which can simultaneously estimate the reliability of annotators as well as the ground-truth of the labels. Our contributions are: 1) a measure of information content in these models, 2) three metrics to estimate expected information gain of new labels, and 3) three general active labelling algorithms that use these metrics to efficiently acquire labels, and give QoI guarantees.

A related active labelling method is proposed in [2], which blacklists less reliable annotators to improve label collection for (semi-)static data-sets. Our approach differs in that we present novel techniques to efficiently collect human labels in dynamic, and time critical crowdsensing applications. We also accomodate different constraints on how labels are sourced, and our information theoretic pairing metrics match annotators with specific tasks, so we can respond to individuals' strengths and weaknesses. Unlike in [2], we do not need to manually set a threshold for reliability of our annotators, instead pairing is performed flexibly and based on need. This means that we can differentiate between rapidly collecting labels of a given quality, or efficiently improving the quality of the data-set as a whole. [18] presents an active labelling approach. However, this requires annotators to state their confidence on labels, and a metric over object descriptors, so it is less broadly applicable.

Other related Bayesian models, e.g. those in [19], [20], model the characteristics of each required label, e.g. difficulty, and these are good candidate models on which to develop extensions of this work. [21] presents lightweight inference for binary semi-trusted labels, but it is unclear how this model could be similarly exploited for active labelling to give the same rich QoI guarantees.

The rest of the paper is laid out as follows: Section II introduces certain methods for inference with semi-trusted 
labels, and outlines a few useful concepts from information theory; Section III details our novel adaptations to these methods for the participatory sensing domains; Section IV tests our methods' performance on real and synthetic data against more conventional approaches; and Section V summarises the findings and discusses future work.

\section{BACKGROUND}

Recent research on crowdsourcing has applied probabilistic models to large scale problems with semi-trusted meta-data, e.g. [1], [2], [21]. These approaches focus on simple labels (meta-data), e.g. binary, categorical, real scalars and vectors, for discrete data items, and aim to simultaneously estimate the hidden ground-truth of labels, and the reliabilities of the annotators. These approaches exploit the principle that reliable annotators tend to agree with each other more often than those prone to random mistakes, bias, or error.

Consider first the basic trust model ${ }^{1}$ outlined in [1]. This assumes there is a set of objects $\mathcal{O}$, which requires annotation, and for each object $i \in \mathcal{O}$ there is an unknown ground-truth $z_{i}$. There is also a set of annotators, $\mathcal{A}$, who can be queried about each $z_{i}$. Each annotator $j \in \mathcal{A}$, is not entirely reliable, and when queried about $z_{i}$ gives label $l_{i j}$, which may, or may not, be equal to $z_{i}$. This model makes the weak assumption that the majority of annotators are non-malicious. If true, then more labels will lead to better inference, as on average even information from weak annotators adds information.

Figure 1 shows the trust model, in which large nodes represent random variables (RVs), and shaded nodes are observed; directed edges show the direction of causal influence; larger rectangles collect groups of RVs; and smaller nodes are the prior parameters of the model. Together, this represents a joint probability distribution. Ground-truths $z_{i}$, labels $l_{i j}$, and annotator reliability estimates, $a_{j}$, are represented in the model by RVs. For simplicity, we focus on binary labels, i.e. $z_{i}, l_{i j} \in\{0,1\}$, but this model can be used with 1-of$K$ categorical, ordinal, real scalar and real vector labels. Here, reliability estimates $a_{j}=\left(a_{j, 0}, a_{j, 1}\right)$, where $a_{j, 0}$ and $a_{j, 1}$ are annotator $j$ 's true negative rate (specificity) and true positive rate (sensitivity) respectively.

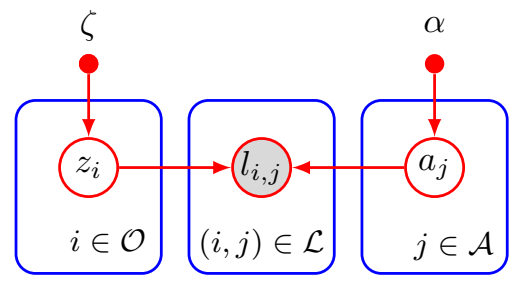

Fig. 1. Simple graphical model to infer ground truth from semi-trusted labels - object $i \in \mathcal{O}$ has hidden ground-truth $z_{i}$, annotator $j \in \mathcal{A}$ has reliability $a_{j}$, and $l_{i j} \in \mathcal{L}$ are observed labels. Parameters $\zeta$ and $\alpha$ encode prior knowledge.

\section{A. Information Entropy and Gain}

Information entropy and gain are used in Section III to anticipate the value of a new label. Information theory [22] tells us that before we know the outcome of some discrete random variable $(\mathrm{RV}), X$, then its entropy is given by

$$
H(X)=\sum_{x \in \mathcal{A}_{X}} P(x) \log _{2} \frac{1}{P(x)}
$$

\footnotetext{
${ }^{1}$ Similar to the model used in [2], where poor labellers are blacklisted.
}

where $\mathcal{A}_{X}$ are possible values for $X$, and $P(x)$ is the probability $X=x$. Put simply, $H(X)$ measures how much information we gain by knowing the outcome of $X$.

For two RVs, $X$ and $Y$, the average entropy remaining in $X$ after knowing $Y$ is given by the conditional entropy

$$
H(X \mid Y)=\sum_{x, y \in \mathcal{A}_{X} \times \mathcal{A}_{Y}} P(x, y) \log _{2} \frac{1}{P(x \mid y)}
$$

where $P(x, y)$ is the joint probability that $X=x$ and $Y=y$, and $P(x \mid y)$ is the probability $X=x$ given $Y=y$.

A related concept is mutual information, which measures the average reduction in entropy of $X$ by knowing $Y$, and is

$$
I(X ; Y)=H(X)-H(X \mid Y)
$$

We define two more information theoretic quantities. First, the joint entropy of $N$ independent RVs $X_{1}, \ldots, X_{N}$ is

$$
H\left(X_{1}, \ldots, X_{N}\right)=\sum_{n} H\left(X_{n}\right)
$$

where independence means $P\left(x_{1}, x_{2} \ldots, x_{N}\right)=\prod_{n} P\left(x_{n}\right)$.

If $\mathrm{RV} X$ has distribution $P$, and we think it has distribution $Q$, then the Kullback-Leibler (KL) divergence measures the information we gain by learning true distribution $P$, and is

$$
D_{\mathrm{KL}}(P \| Q)=\sum_{x \in \mathcal{A}_{X}} P(x) \ln \left(\frac{P(x)}{Q(x)}\right)
$$

Bayesian probabilistic models allow us to treat beliefs about unknown quantities as probability distributions over RVs. Together with the above information theoretic quantities, this concept allows us to measure how changing beliefs correspond to information gains. In the next section, we show how these measures can be used to guide knowledge acquisition.

\section{ACtive LABEL COLlection}

This paper considers labels about objective reality, e.g. map locations; or about a shared view, e.g. whether a cycle route is challenging. Given this, the object being labelled must be experienced. We categorise labels as either singular experience or repeatable experience. Singular experience labels can only be acquired contemporaneously with the data, because the original experience cannot be sufficiently reconstructed from the data to be re-experienced. For example, the categorisation of human movement, e.g. cycling or walking, from accelerometer and gyroscope readings. Repeatable experience labels are such that a human inspecting or experiencing the data can reasonably be expected to label it. A trivial example is labelling an image based on its content. However, other examples exist, such as deciding whether there is traffic noise or human conversation in a sound recording (applicable to [17] and [5] respectively); or tagging the name and function of a map location (used in [5], [7], [8]). One common form of repeatable experience labelling is the up-/down-voting of data (or meta-data), e.g. suggested cycle routes [17], or the location of pot-holes [14]. This paper focuses on labels corresponding to repeatable experience, as the modelling techniques we employ require multiple labels for data items. More precisely, they require a degree of overlap in data items labelled by different annotators. 


\section{A. Object versus Annotator Centricity}

Crowdsourcing (and hence crowdsensing) annotation applications come in many forms, and with different ways in which data items (more generally tasks) are selected for annotators, and how annotators are notified of requests, called staging. Figure 2 shows two common approaches. The first is annotator centric staging, see Figure 2(a), where annotators are central to the task assignment, and objects are selected, either individually or in batches, and queued for each annotator. This approach is common for the labelling of large data-sets, where annotators may attend sessions of annotation. In object centric staging, see Figure 2(b), tasks arrive-or are selected in turnand are then assigned to a waiting crowd of annotators. This approach is suited to live, on-going annotation, such as you might find in crowdsensing applications, where annotators are on call to answer questions for certain periods of the day.

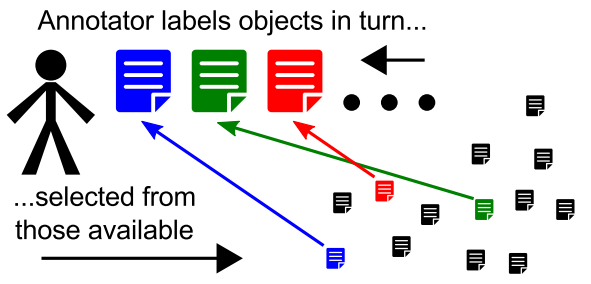

(a) Annotator centric staging

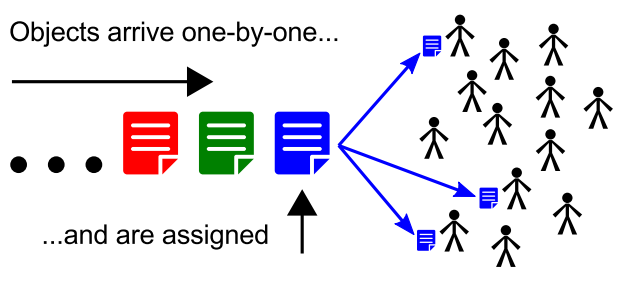

(b) Object/task centric staging

Fig. 2. Annotator centric versus object/task centric staging.

There are a number of additional concerns that can apply to both approaches, e.g. it may be that:

- Ground-truth corresponds to objective reality, or to hypothetical consensus, i.e. population majority view.

- The ground-truth is used as training inputs to learning algorithms, either for a single known algorithm or as a general purpose data-set.

- There is marginal utility to improving the confidence in ground-truth predictions beyond a threshold.

- QoI is either associated with the number of objects above the confidence threshold, or more generally with overall confidence measured across all objects.

- Some objects require higher confidence than others.

This paper focuses on general purpose active labelling for objective ground-truth, treats all objects as equal in terms of information quality, and explores methods to improve both confidence threshold and overall confidence metrics. As stated in [1], there is no fundamental difference in the treatment of objective ground-truth and hypothetical consensus, so we approach both label types in the same way.

\section{B. Object Centric Staging}

Object centric labelling involves finding the right annotators for a given object or task. For this, the central authority must be able to request labels from individuals. There are a number of additional concerns associated with object centric approaches. For example, the central authority may wish to:

- select one annotator for each object, or more than one.

- account for annotators failing to respond to requests, for annotators to respond at different rates, or with response rates that change with time

- limit the number of responses per object a priori by number, or reactively based on information measures.

- associate a higher cost with labels from some annotators, or with waiting longer for a response.

We focus on assigning one annotator per object at a time, assume that all annotators respond reasonably quickly, and allow costs to be a balance between time and number of labels. Other complicating factors are considered straightforward extensions of the methods here. We explore object centric approaches first and propose two general staging algorithms.

Buffered Streaming: A small number of objects/tasks are queued in a buffer. Iteratively, the queue's head is assessed, and if the inference is still unsatisfactory, it is paired with an annotator and marked awaiting; otherwise the object is done (qualified). When a an awaiting label is returned (or times out), the object is moved to to the back of the queue.

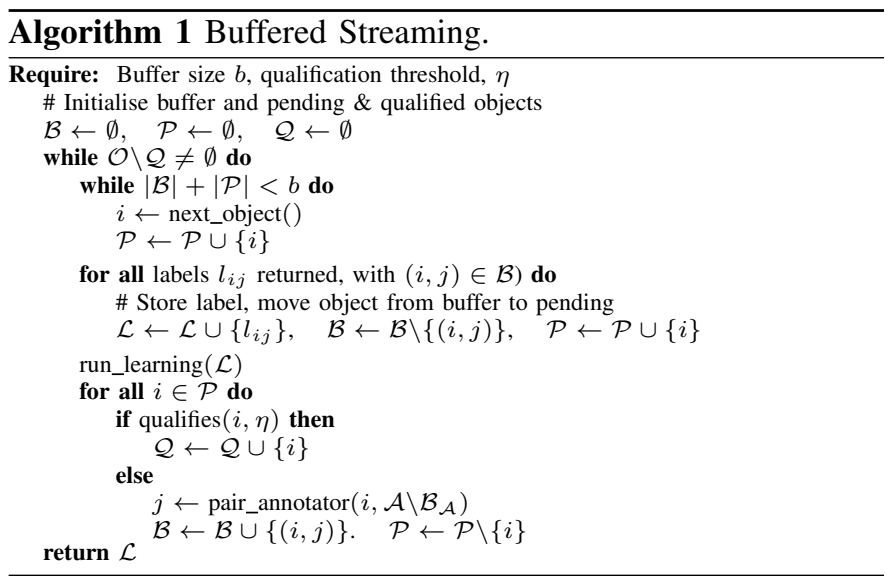

Buffered Pool Sampling: Objects are kept together in a pool, then sampled individually from the pool, either randomly or based on inference. Sampled objects are allocated to a annotator, and pushed to a finite awaiting buffer. Objects exit the awaiting buffer when response is received (or times out). As new responses become available, inference can be rerun, and satisfactory objects marked as qualified.

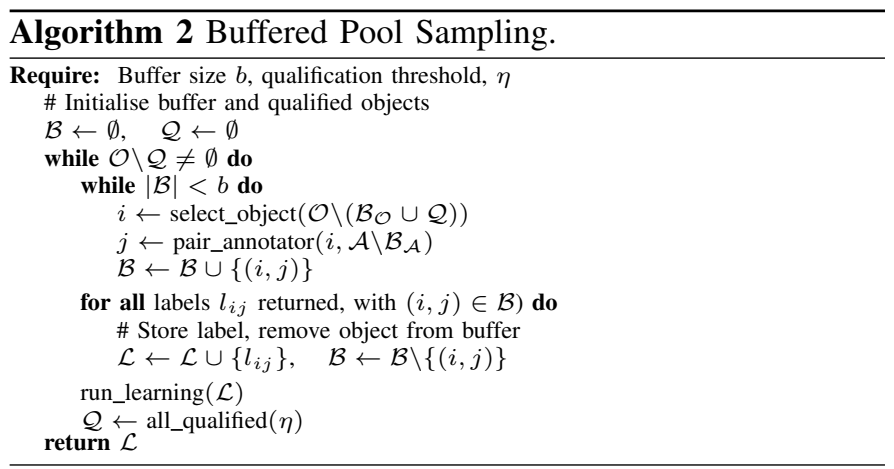

Buffered streaming and buffered pool sampling are outlined in Algorithms 1 and 2 respectively. In both algorithms, the set $\mathcal{A}$ is the current set of annotators on call, and annotators 
may dynamically join and leave this set. The set $\mathcal{O}$ contains all objects recognised by the system. In general, new objects will be added to (or possibly removed from) this set with time. Busy annotators are defined as those in a buffer $\mathcal{B}_{\mathcal{A}} \stackrel{\text { def }}{=}\{j \mid(i, j) \in \mathcal{B}\}$, and objects that have been assigned and are awaiting responses are defined as $\mathcal{B}_{\mathcal{O}} \stackrel{\text { def }}{=}\{i \mid(i, j) \in \mathcal{B}\}$. next_object () is used by buffered streaming to schedule the next object for labelling. Most simply, this is done by timestamp or sampled at random. However, active-learning could be used when coupled with a classification/regression algorithm, see Section V. run_learning $(\mathcal{L})$ takes the current labels $\mathcal{L}$, and runs the inference, giving new ground-truth and reliability estimates. qualifies $(i, \eta)$ determines if the inference for object $i$ is sufficiently certain, and takes a threshold, $\eta$, as argument - we discuss this further in Section III-D. all_qualified $(\eta)$ simply returns the set of all sufficiently certain objects, i.e. $\{i \in \mathcal{O} \mid$ qualifies $(i, \eta)\}$. pair_annotator $\left(i, \mathcal{A} \backslash \mathcal{B}_{\mathcal{A}}\right)$ predicts the best available annotator to label object $i$, and select_object $(\overline{\mathcal{O}})$ is used by buffered pool sampling to schedule the next object, both are discussed further in Section III-D. Buffered streaming is clearly more constrained than buffered pool sampling, the latter allowing the central authority to choose objects for every pairing based on changing need but with an additional overhead. However, buffered streaming ensures that each object qualifies in turn, and so can be used to rapidly acquire individual objects of a given quality.

\section{Annotator Centric Staging}

Annotator centric labelling involves choosing appropriate objects for users who annotate in sessions. Annotator centric approaches may additionally wish to:

- allow annotators to terminate sessions at any point.

- have many annotators in session at any time.

- account for annotators working more or less quickly, with response times that depend on time and task.

We propose one annotator centric staging algorithm, which supports multiple annotators, who may join or terminate sessions freely. We assume response times are probabilistic but, for simplicity, assume these do not depend on task, time or annotator. Suitable extensions are straightforward.

Session Queues: Each in session annotator is assigned a queue of up to $n$ objects. At each iteration, annotators are assessed individually and suitable objects are allocated to return queues to size $n$. As annotators leave sessions, queues are cleared, and are freshly filled to size $n$ when they rejoin.

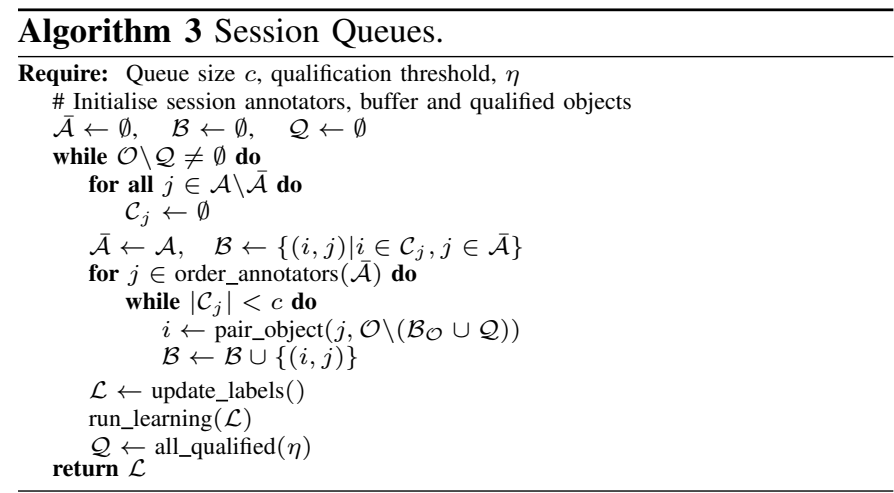

Session Queues staging is outlined in Algorithm 3, where in-session annotators are denoted $\overline{\mathcal{A}}$, and update_labels() adds all newly returned labels to $\mathcal{L}$. Finally, order_annotators $(\overline{\mathcal{A}})$ determines what order the in session annotators will have their queues filled, and pair_object $\left(j, \mathcal{O} \backslash\left(\mathcal{B}_{\mathcal{O}} \cup \mathcal{Q}\right)\right)$ predicts the best unqueued object for annotator $j$ to label, again, both are discussed further in Section III-D.

\section{Information and Pairing Metrics}

As discussed in Section II, the information content of probabilistic beliefs (or rather the degree of uncertainty) can be measured using the entropy of the corresponding random variable (RV). Here we show how this relates to the simple trust model from Section II for binary labels ${ }^{2}$, and how we use this to mark objects as satisfied and pair them with annotators. Recall that for labels $\mathcal{L}$, the trust model infers ground-truth $z_{i}$ for each object $i$. For our binary model, $z_{i} \in\{0,1\}$, and this unknown outcome is modelled by a RV, $Z_{i}$, where

$$
P\left(z_{i}=z \mid \mathcal{L}\right)=\left(1-\mu_{i}\right)^{(1-z)} \mu_{i}^{z}
$$

For notational simplicity, we drop the explicit label condition, i.e. $P\left(z_{i} \mid \mathcal{L}\right)=P\left(z_{i}\right)$. Given this represents a probability distribution over possible ground-truths $z_{i}$, we can measure the entropy of $Z_{i}$, using Equations (1) and (6), to give

$$
H\left(Z_{i}\right)=-\left(1-\mu_{i}\right) \log \left(1-\mu_{i}\right)-\mu_{i} \log \left(\mu_{i}\right)
$$

Each label $l_{i j}$ we have not yet collected also represents a RV $L_{i j}$. We can again calculate the entropy $H\left(L_{i j}\right)$, by noting

$$
\begin{aligned}
& P\left(l_{i j}=l\right)=\sum_{z} P\left(l_{i j}=l \mid z_{i}=z\right) P\left(z_{i}=z\right) \quad \text { and } \\
& P\left(l_{i j}=l \mid z_{i}=z\right)=a_{j, l}^{\mathcal{I}[l=z]}\left(1-a_{j, l}\right)^{(1-\mathcal{I}[l=z])}
\end{aligned}
$$

where $\mathcal{I}[$.$] is the indicator function.$

From Equation (4), the total entropy over all objects is

$$
H(\mathcal{O})=\sum_{i \in \mathcal{O}} H\left(Z_{i}\right)
$$

because conditioned on the $\mu_{i} \mathrm{~s}$ the $Z_{i} \mathrm{~s}$ are independent.

Information theory states that new data will on average add information [22], and hence decrease this entropy. Our aim is to select a new label $l_{i j}$ that will most reduce $H(\mathcal{O})$. In our model, $L_{i j}$ has a more direct relationship to $Z_{i}$, than other $Z_{i^{\prime}} \mathrm{s}$, therefore we assume that choosing $i$ and $j$ to maximally reduce $H\left(Z_{i}\right)$ is a good substitute strategy. This is not a formal argument. We present three pairing metrics, $P M_{i, j}$, that predict the information gain in $Z_{i}$ given $l_{i j}$.

Mutual Information (MI): If we knew no more about $Z_{i}$ and $L_{i j}$, than their joint probability, then the mutual information between $Z_{i}$ and $L_{i j}$ tells us how much new information about $Z_{i}$ is acquired when we know $l_{i j}$. From Equations (2) and (3), this is

$$
P M_{i, j}=I\left(L_{i j} ; Z_{i}\right)=H\left(L_{i j}\right)-\sum_{z_{i}, l_{i j} \in\{0,1\}} P\left(l_{i j} \mid z_{i}\right) P\left(z_{i}\right) \log \left(\frac{1}{P\left(l_{i j} \mid z_{i}\right)}\right)
$$

and can be evaluated using Equations (6), (7) and (8).

Note that $L_{i j}$ and $Z_{i}$ are non-trivially coupled by the inference model, so this may not be a perfectly accurate measure. However, it is a good first order approximation.

\footnotetext{
${ }^{2}$ It is straightforward to extend this approach to other kinds of labels.
} 
Information Gain $(I G)$ : Alternatively, we can predict how estimates of $z_{i}$ change, if we acquire $l_{i j}$. Define $Z_{i}^{\prime}$ as new probability distribution that would be inferred if $l_{i j}$ were added to $\mathcal{L}$. More specifically, if $l_{i j}=l$ then $Z_{i}^{\prime}=\left.Z_{i}\right|_{l_{i j}=l}$. The information gained by adding $l_{i j}=l$ is then estimated by $D_{\mathrm{KL}}\left(Z_{i}\left|l_{i_{j}=l}\right| \mid Z_{i}\right)^{3}$. As we do not know the label in advance, we calculate the expected information gain

$$
\begin{aligned}
P M_{i, j} & =\mathbf{E}\left(D_{\mathrm{KL}}\left(Z_{i}^{\prime}|| Z_{i}\right) \mid l_{i j}\right)=\sum_{l_{i j} \in\{0,1\}} P\left(l_{i j}\right) D_{\mathrm{KL}}\left(\left.Z_{i}\right|_{l_{i j}}|| Z_{i}\right) \\
& =\sum_{z_{i}, l_{i j} \in\{0,1\}} P\left(l_{i j} \mid z_{i}\right) P\left(z_{i}\right) D_{\mathrm{KL}}\left(\left.Z_{i}\right|_{l_{i j}}|| Z_{i}\right)
\end{aligned}
$$

using Equations (6) and (8) and run_learning $\left(\mathcal{L} \cup\left\{l_{i j}\right\}\right)$.

Thresholding (T): The third pairing metric, requires a threshold $\eta$, and is the probability that $l_{i j}$ will increase object $i$ 's entropy beyond threshold $\eta$, e.g. $P\left(H\left(Z_{i}^{\prime}\right)>\eta\right)$. This again uses run_learning $\left(\mathcal{L} \cup\left\{l_{i j}\right\}\right)$ and is given by

$$
P\left(H\left(Z_{i}^{\prime}\right)>\eta\right)=\sum_{l_{i j} \in\{0,1\}} P\left(l_{i j}\right) \mathcal{I}\left[H\left(\left.Z_{i}\right|_{l_{i j}}\right)>\eta\right]
$$

Approximating $Z_{i}^{\prime}$ : Note that information gain and thresholding's use of run_learning $\left(\mathcal{L} \cup\left\{l_{i j}\right\}\right)$ can be expensive. However, methods from [1] and [2] use expectationmaximisation algorithms which iteratively approximate all $\mu_{i}$ and $a_{j}$ simultaneously. For models already trained on moderately sized label sets, adding one label and retraining is relatively cheap, and the vast majority of the change in $Z_{i}$ occurs in the first E-step. For these reasons, we use a single E-step approximation of each $Z_{i}^{\prime}$, in our experiments.

\section{E. Selection, Qualification and Pairing}

The above measures are used as follows:

select_object $(\overline{\mathcal{O}})$ : in buffered pool sampling, schedules the next object given those available, $\mathcal{O}$. This selection can take advantage of the entropy of ground-truth predictions, and we examine three possibilities: random, object chosen at random; most certain, choose object $i^{\star}=\operatorname{argmax}_{i \in \overline{\mathcal{O}}} H\left(Z_{i}\right)$; and least certain, choose object $i^{\star}=\operatorname{argmin}_{i \in \overline{\mathcal{O}}} H\left(Z_{i}\right)$.

qualifies $(i, \eta)$ : determines if the inference for object $i$ is sufficiently certain given scalar threshold $\eta>0$. For generality, this uses the entropy as a measure of certainty, i.e. returning $H\left(Z_{i}\right) \leq \eta$. For binary labels, this is equivalent to a probability threshold $\eta^{\prime}$, where sufficient certainty corresponds to $\mu_{i} \leq \eta^{\prime}$ or $\mu_{i} \geq\left(1-\eta^{\prime}\right)$; asymmetric thresholds are also possible.

pair_object $(j, \overline{\mathcal{O}})$ : finds the best object $i^{\star}$, for annotator $j$ from those available. This uses any pairing metric, $P M_{., j}$, and returns $i^{\star}=\operatorname{argmax}_{i \in \overline{\mathcal{O}}} P M_{i, j}$.

pair_annotator $(i, \overline{\mathcal{A}})$ : finds the best annotator, $j^{\star}$, for object $i$ from those available, again based on a pairing metric, $P M_{i}$., where $j^{\star}=\operatorname{argmax}_{i \in \overline{\mathcal{A}}} P M_{i, j}$.

order_annotators $(\overline{\mathcal{A}})$ : orders annotators for processing by session queues. We explore three possibilities: Egality (E), i.e. random order; Meritocracy (M), i.e. by decreasing reliability; and Cannon Fodder (C), i.e. by increasing reliability.

\footnotetext{
${ }^{3}$ To see why, note that $\left.Z_{i}\right|_{l_{i j}=l}$ would then be our best approximation of the ground-truth.
}

pair_annotator(.,.) and pair_object(.,.), also support random (passive) pairing. However, the staging methods discussed here are only truly effective, with non-passive (active) pairing.

\section{RESUlTS}

The data-set most closely satisfying our experimental requirements was the Cub200 birds [2]. However, this has too little overlap between annotators, and no ground-truth. To address this, we trained the inference model on real labels for an individual binary Cub200 attribute, e.g. has_size::small, then sampled 500 object ground-truths and 50 annotator expertises from the inferred values, to synthetically generate labels with known ground-truth. Response times were sampled from an exponential distribution with mean of 1 minute.

Figure 3(a) shows final label count, time, information entropy and ground-truth error rate for 5 runs of each object centric approach, as well as naive label collection. . Results show buffered streaming terminates more quickly than naive sampling and with just over half as many labels, while the best pool sampling method takes $\sim 50 \%$ longer, but again with fewer labels. Note that performance for all methods have error rates slightly higher than their thresholds, with buffered streaming as least accurate. Figure 3(b) shows how error rate threshold affects buffered streaming performance, with stricter thresholds reducing the number of labels (cost), but with increased time penalty. Mutual information pairing leads to fewest labels, while Thresholding gives the best accuracy.
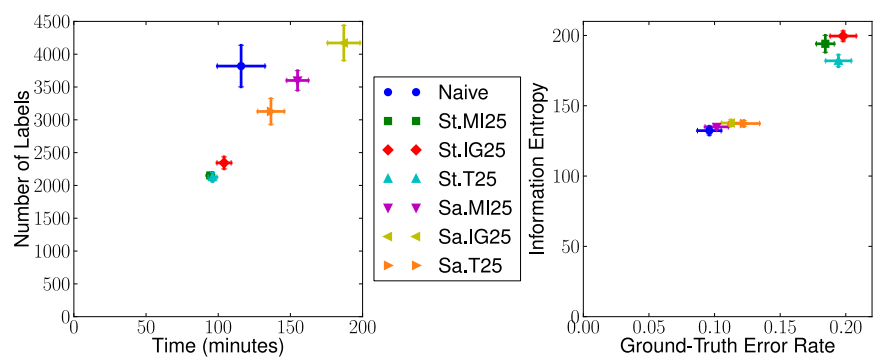

(a) Streaming (St.) and pool sampling (Sa.); threshold error rate 0.1.
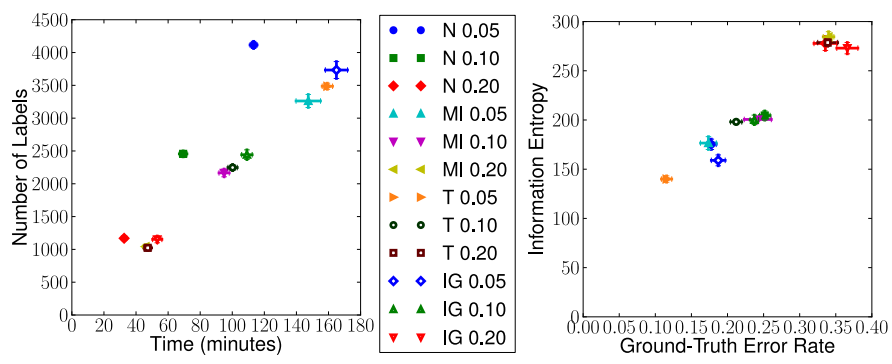

(b) Buffered streaming, variable threshold error shown in legend.

Fig. 3. Object centric results, with pairing metrics mutual information (MI), information gain (IG) and threshold crossing (T). Buffer size was 25 for active methods. Naive $(\mathrm{N})$ method also shown. Error bars are 1 std. error.

Figure 4 (a) and (b) show the effects of varying ordering and queue length on the session queues staging respectively, where annotators join/leave sessions with probability 0.1 each iteration. Each experiment represents 5 runs for a fixed 50000 iterations, so only accuracy and entropy results are shown. Figure 4 (a), shows Egality ordering, with thresholding and information gain metrics, leads to higher accuracy than all 
other choices. Figure 4 (b) uses Egality ordering and shows that larger queues give slightly higher throughput of labels.

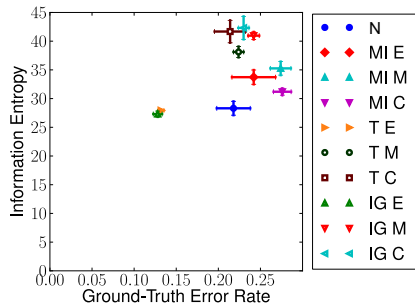

(a) Annotator order

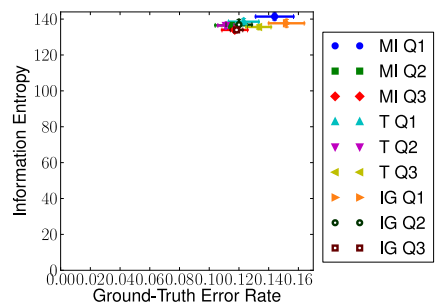

(b) Session Queues
Fig. 4. Session queues, with pairing metrics: Mutual Information (MI), Thresholding (T) and Information Gain (IG). (a) compares (E)gality, (M)eritocracy, and (C)annon Fodder ordering, and (b) compares different Queue lengths $n$, denoted $\mathrm{Q} n$. Error bars are 1 std. error.

\section{Conclusion}

This paper outlines a family of approaches for active labelling within crowdsensing systems. These methods are based on information theoretic principles and cater for a variety of constraints on how labels are collected, i.e. staging. Object centric staging offers ways in which the number of labels required can be significantly reduced with small or no impact on time, and this will be particularly relevant when there is a cost associated with label collection. However, there are additional benefits, including a finer grained control over label collection, and cleaner data-sets, e.g. a smaller number of low quality labels. Unsurprisingly the naive approach is competitive on time though, as it allows maximal throughput of information, every annotator providing labels at their maximum rate. Annotator centric staging shows a marked improvement in accuracy over naive approaches for fixed label collection time, and labels can be queued in advance for each annotator. Both staging approaches can improve the acquisition rate and accuracy of inferred ground truth.

Aggressively optimising label acquisition could, in theory, lead to reduced accuracy due to confirmation bias, i.e. seeking to confirm what one already believes. However, there was no evidence of this in our results. The entropy measure correlates well with the error rate for all of our algorithms.

In future work, we intend to support other label types \& QoI measures (e.g. those in [23]); to account for time, task $\&$ annotator dependent response rates, and easier \& harder tasks; and perform case studies on real collection in the wild. Another research avenue is to account for the specific learning algorithms that use the data, by integrating active label collection with active learning. The general principles outlined here offer a strategic base from which to explore these ideas.

\section{ACKNOWLEDGMENT}

This work was funded by EIT ICT Labs under the City Crowd Source Activity (13064). We are also grateful to Dr. Anil Bharath at Imperial, and to colleagues within the project, for the many useful discussions which influenced this work.

\section{REFERENCES}

[1] V. C. Raykar, S. Yu, L. H. Zhao, A. Jerebko, C. Florin, G. H. Valadez, L. Bogoni, and L. Moy, "Supervised Learning from Multiple Experts: Whom to Trust when Everyone Lies a Bit," in ICML, 2009, pp. 889896.
[2] P. Welinder and P. Perona, "Online crowdsourcing: rating annotators and obtaining cost-effective labels," in CVPR, 2010, pp. 2262-2269.

[3] D. Mendez and M. A. Labrador, "On Sensor Data Verification for Participatory Sensing Sys.” J. of Networks, vol. 8, pp. 576-87, 2013.

[4] M. Riahi, T. G. Papaioannou, I. Trummer, and K. Aberer, "Utility-driven data acquisition in participatory sensing," in Proc. of 16th Intl. Conf. on Extending Database Technology, ser. EDBT '13, 2013, pp. 251-262.

[5] E. Miluzzo, N. D. Lane, K. Fodor, R. Peterson, H. Lu, M. Musolesi, S. B. Eisenman, X. Zheng, and A. T. Campbell, "Sensing meets mobile social networks: the design, implementation and evaluation of the cenceme application," in Proc. of 6th ACM Conf. on Embedded Network Sensor Sys., 2008, pp. 337-50.

[6] A. Stopczynski, J. Larsen, S. Lehmann, L. Dynowski, and M. Fuentes, "Participatory bluetooth sensing: A method for acquiring spatiotemporal data about participant mobility and interactions at large scale events," in Pervasive Computing and Comms. Wksps. (PERCOM), 2013.

[7] C. S. Karjalainen, "Moves: Activity Tracking without Gadgets," 2013, accessed on: 23/10/2013. [Online]. Available: moves-app.com

[8] N. Bulusu, C. T. Chou, S. Kanhere, Y. Dong, S. Sehgal, D. Sullivan, and L. Blazeski, "Participatory Sensing in Commerce: Using Mobile Camera Phones to Track Market Price Dispersion," in Wksp. on Urban, Community, and Social Apps. of Networked Sensing Sys., 2008.

[9] L. Deng and L. P. Cox, "Livecompare: grocery bargain hunting through participatory sensing," in Proc. of 10th Wksp. on Mobile Computing Sys. and Applications, 2009, pp. 4:1-4:6.

[10] S. Devarakonda, P. Sevusu, H. Liu, R. Liu, L. Iftode, and B. Nath, "Realtime air quality monitoring through mobile sensing in metropolitan areas," in Proc. of 2nd ACM SIGKDD Intl. Wksp. on Urban Computing, ser. UrbComp '13, 2013, pp. 15:1-15:8.

[11] E. D'Hondt, M. Stevens, and A. Jacobs, "Participatory noise mapping works! an evaluation of participatory sensing as an alternative to standard techniques for environmental monitoring," Pervasive and Mobile Computing, vol. 9, no. 5, pp. $681-694,2013$.

[12] K. Han, E. A. Graham, D. Vassallo, and D. Estrin, "Enhancing motivation in a mobile participatory sensing project through gaming," in SocialCom/PASSAT, 2011, pp. 1443-1448.

[13] J. Burke, D. Estrin, M. Hansen, A. Parker, N. Ramanathan, S. Reddy, and M. B. Srivastava, "Participatory Sensing," in Wksp. on WorldSensor-Web, 2006, pp. 117-134.

[14] J. Eriksson, L. Girod, B. Hull, R. Newton, S. Madden, and H. Balakrishnan, "The Pothole Patrol: Using a Mobile Sensor Network for Road Surface Monitoring," in Mobile Sys., Apps. and Services, 2008.

[15] U. Lee, B. Zhou, M. Gerla, E. Magistretti, P. Bellavista, and A. Corradi, "Mobeyes: smart mobs for urban monitoring with a vehicular sensor network," Wireless Comms., IEEE, vol. 13, no. 5, pp. 52-57, 2006.

[16] W. Sha, D. Kwak, B. Nath, and L. Iftode, "Social vehicle navigation: integrating shared driving experience into vehicle navigation," in Proc. of 14th Wksp. on Mobile Computing Sys. and Apps., 2013, pp. 16:1-6.

[17] S. Reddy, K. Shilton, G. Denisov, C. Cenizal, D. Estrin, and M. Srivastava, "Biketastic: sensing and mapping for better biking," in SIGCHI Conf. on Human Factors in Computing Sys., 2010, pp. 1817-1820.

[18] E. A. Ni and C. X. Ling, "Active learning with c-certainty," in Proc. of 16th Pacific-Asia Conf. on Advs. in Knowledge Discovery and Data Mining, 2012, pp. 231-242.

[19] P. Welinder, S. Branson, S. Belongie, and P. Perona, "The multidimensional wisdom of crowds," in NIPS, 2010, pp. 2424-2432.

[20] J. Whitehill, P. Ruvolo, T. Wu, J. Bergsma, and J. Movellan, "Whose vote should count more: Optimal integration of labels from labelers of unknown expertise," in NIPS, 2009, pp. 2035-2043.

[21] X. Liu, L. Li, and N. Memon, "A lightweight combinatorial approach for inferring the ground truth from multiple annotators," in Mach. Learn. and Data Mining in Pattern Recog., 2013, vol. 7988, pp. 616-628.

[22] D. J. C. MacKay, Information Theory, Inference \& Learning Algorithms. New York, NY, USA: Cambridge University Press, 2004.

[23] C. Liu, P. Hui, J. Branch, and B. Yang, "Qoi-aware energy management for wireless sensor networks," in Pervasive Comp. and Coms. Workshops (PERCOM Workshops), 2011, pp. 8-13. 